\title{
The role of midwives in improving breastfeeding practices in protracted crises: The Gaza Strip example
}

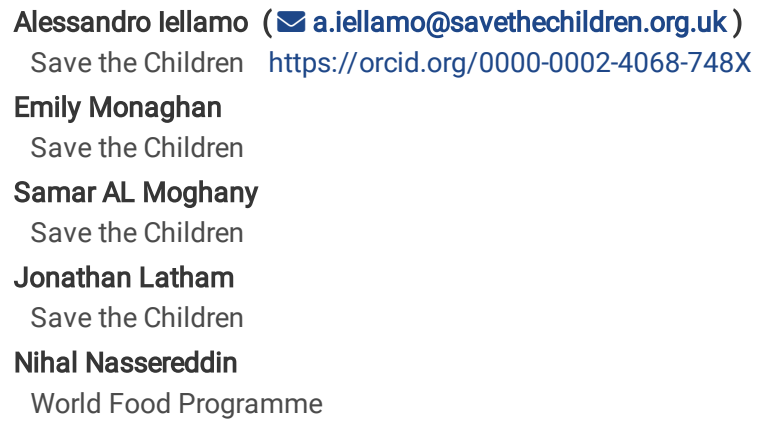

Research in practice

Keywords: Breastfeeding, Breastfeeding in emergencies, IYCF, IYCF-E, New-born care, Early initiation of breastfeeding, Infant feeding in emergencies, Midwife, Counselling, BFHI

Posted Date: May 20th, 2020

DOI: https://doi.org/10.21203/rs.3.rs-22328/v1

License: (c) (i) This work is licensed under a Creative Commons Attribution 4.0 International License. Read Full License

Version of Record: A version of this preprint was published at BMC Public Health on April 17th, 2021. See the published version at https://doi.org/10.1186/s12889-021-10748-2. 


\section{Abstract}

\section{Background:}

The protection, promotion and support of breastfeeding is the most effective intervention to prevent child morbidity and mortality in all contexts.

During the Palestine-Israel conflict that started in the middle of the $20^{\text {th }}$ century, healthcare services are understaffed and lack basic resources, with frequent power cuts and stock-outs of essential drugs and equipment. This case study seeks to answer the questions: (1) How does the protracted crisis in Gaza affect the breastfeeding practices of the most vulnerable population; and (2) What is the role that midwives can play in improving breastfeeding practices?

A mixed method (qualitative and quantitative) study was used to collect data. A total of 1044 respondents had a child less than 24 months at the time of the study. A total of 62.75 percent practice early initiation of breastfeeding and 42 percent confirmed that their new-borns were given liquids other than breast milk during the first three days of life. Fifty percent of mothers addressed breast milk insufficiency by drinking additional fluids and 40 percent by using infant formula.

\section{Discussion:}

This study confirms the need to a) implement the IYCF-E operational guidance in the Gaza strip and b) review the pre-service and in-service training for midwives. For vulnerable populations in humanitarian contexts, the impact of this cannot be underestimated.

Conducting research in humanitarian settings is particularly challenging. Our experience included integration and linkage with international organizations security officers on the ground, co-ordination of activities with authorities and communities, regular supportive supervision during data collection, allocation of buffer days and geographical areas and in-country and remote assistance to data collection teams

\section{Conclusions:}

This research focuses on a protracted emergency in insecure populated areas. The unpredictable security situation meant that the team adopted a flexible approach during data collection. Lessons learned throughout this process include the importance of a) allocating additional research time, to account for potential interruption b) daily coordination with security officers to ensure safe access to localities c) identification of extra sites, in case escalation of conflict d) training of additional enumerators in case conflict escalation e) negotiation with authorities to ensure compliance with requirements.

\section{Background}

\subsection{Humanitarian Context}

In the Gaza Strip, ten years of blockade imposed by Israel after the takeover of Gaza by Hamas, and further recurrent outbreaks of hostilities have eroded basic infrastructure, service delivery, livelihoods and coping mechanisms. In 2019, the United Nations Office for the Coordination of Humanitarian Affairs (UNOCHA) suggested that issues affecting vulnerability in occupied Palestinian territory (oPt) include: the risk of and/or exposure to violence; the policies and restrictions imposed on geographic locations; and the specific contextual vulnerabilities that arise as a result.(1) The chronic electricity deficit has severely affected the availability of essential services, particularly health, water and sanitation services, and undermined Gaza's fragile economy, particularly the manufacturing and agriculture sectors.(1) Palestinians in the Gaza Strip without access to safe water or sanitation have been identified among the most vulnerable groups in need of humanitarian assistance. (1) Instability, repeated and unpredictable escalation of conflict and limitation of movement for international and sometimes national experts, poses significant challenges to the ability of national and international agencies to conduct quality and consistent research.

The health sector has been heavily disrupted by years of conflict, sanctions and socio-economic decline. (3) Healthcare services are understaffed and lack basic resources, with frequent power cuts and stock-outs of essential drugs and equipment. (4) Over $90 \%$ of the water is unsuitable for human consumption. (3) Psychological trauma and poverty have severely affected the population's mental health, with many people, including children and pregnant and lactating women, suffering from anxiety and depression.(3) Less than 50 percent of infants under six months are exclusively breastfeeding. (5). The level of micronutrient deficiency is high; 75 percent of children under 1 year are anaemic and more than 30 percent of pregnant and lactating women suffering from anaemia as well. (6)

\subsection{Research Study}

This case analysis highlights a component of a larger nutrition multisectoral assessment conducted in the Gaza strip and focuses on the following questions:

1) How does the protracted crisis in Gaza affect the breastfeeding practices of the most vulnerable population; and (2) What is the potential role that midwives can play in improving breastfeeding practices?

Data was collected using a mixed method approach with both quantitative and qualitative methods. This included a household survey comprised of anthropometric measurements, a Knowledge, Attitude and Practices (KAP) and an household infant and young child feeding (IYCF) survey, focus group discussions with primary caregivers and key informant interviews with stakeholders. 
The assessment took place across all five governorates of Gaza; poverty data from 2018 was only available at the governorate and not at the locality level. (7). Proxy indicators correlated with for poverty from the 2018 census were identified as household size and highest educational attainment. (7)

In order to collect the sample sizes for the household survey a two-pronged approach to sampling was used. The sample size was calculated a design effect of 1.5 and a 10 percent estimate of non-response. The sample size was calculated based on achieving statistical confidence for the breastfeeding indicators.

Systematic random sampling was carried out by beginning at the administrative edge of the selected area and counting every 10 households to screen for eligibility criteria. If a household was found to have multiple eligible women, a maximum of two women were sampled per household. For each woman, a maximum of two children were eligible for sampling. Purposeful selection of women and children was conducted utilising eligibility and prioritisation criteria.

The household questionnaire was developed in Excel and uploaded onto mobile data collection tool (Kobo) for testing and shared with relevant stakeholders for testing and review.

The approval for the conduct of the assessment was provided by the Ministry of Interior in the Gaza strip, that reviewed, commented and requested changes in the questionnaire and in the design, no other approval was needed by the local authorities to conduct the study.

All the respondents were asked if they agreed to participate in the survey. Their verbal answer was recorded in the Kobo survey tool. The Ministry of Interior reviewed the questionnaire and the methodology including the verbal approval by the respondents to participate in the study.

During the actual implementation of the study, two (2) initially selected areas could not be accessed due to rapid deterioration in the security situation and associated risks of conflict escalation with aerial attacks from both parties involved. Data collection was therefore stopped for 2 days due to the imminent risk of escalation between the parties. Clearance was provided by the security officer prior to the re-start of data collection.

The findings illustrate the level of breastfeeding knowledge and practices of mothers living in poor communities, suggesting an opportunity to increase the ability of the midwives in the Gaza strip to promote, protect, and support breastfeeding during ante-natal, intra-partum and the post-natal period. A total of 1476 respondents (mothers) of children (0-59 months) were covered by the assessment, and all (100\%) delivered in a health facility. 1172 ( $80.7 \%$ ) delivered vaginally, while $284(19.2 \%)$ delivered via a Caesarean-section (CS). A total of 1044 had a child less than 24 months (70.7\%) while the remaining had children 2 to less than 5 years of age.

Findings show that in the context of the Gaza strip, $88 \%$ of women know that breastfeeding should be initiated within the first hour of birth (Table 1 ).

Table 1

Knowledge on when to start breastfeeding

\begin{tabular}{|lll|}
\hline When should you start breastfeeding? & No & (\%) \\
\hline Immediately/within one hour & 919 & 87.77 \\
\hline Within one day & 87 & 8.31 \\
\hline Within two days & 23 & 2.20 \\
\hline When the mother is ready & 12 & 1.15 \\
\hline When the baby wants & 3 & 0.29 \\
\hline Don't know & 2 & 0.19 \\
\hline After three days & 1 & 0.10 \\
\hline
\end{tabular}

More than $50 \%$ of women said that they received most breastfeeding information during antenatal care visits. Only $18 \%$ of women said that they received breastfeeding information during contact with health professionals throughout labour, delivery and subsequent post-natal care visits.

However, despite women's knowledge, this study revealed that $6.3 \%$ of mothers with children less than 24 months stated that they never breastfed their children. When asked about the reasons for never breastfeeding their children, the top five (5) reasons given were: 1) maternal illness (40.3\%); 2 ) new-born illness $(26.9 \%)$; 3 ) baby's refusal to breastfeed (16\%); 4) perceived no/insufficient breast milk (9\%); and 5) preterm baby (4.5\%) or CS delivery (4.5\%).

Early initiation of breastfeeding (breastfeeding within the 1 st hour of birth) was practiced by $62.75 \%$ of mothers of children aged $0-23$ months; $42 \%$ of the respondents confirmed that their new-borns were given liquids other than breast milk during the first three days of life.

Mothers were asked about the way that they addressed breastfeeding complications, such as perceived breast milk insufficiency. More than $50 \%$ answered that they rely on drinking additional fluids, $40 \%$ started to use breast milk substitutes such as infant formula, $21 \%$ of women increased the frequency of breastfeeding and $3 \%$ sought counselling support (Table 2).

Focus group discussions conducted with women affected by the conflict confirm quantitative findings. Qualitative findings show a very high awareness of recommended breastfeeding practices, but the concerns and worries about the current economic situation, coupled with misinformation currently affect the breastfeeding practices in these communities. 
Table 2

Respondents' solutions to perceived breast milk insufficiency

\begin{tabular}{|lll|}
\hline What should you do if you have insufficient breast milk?) & No. & (\%) \\
\hline Drink more fluids & 533 & $51 \%$ \\
\hline Top up each breastfeed with a bottle of formula & 431 & $41 \%$ \\
\hline Increase frequency of breastfeeding & 220 & $21 \%$ \\
\hline Unsure / Don't know & 141 & $13.5 \%$ \\
\hline Seek advice/assistance with positioning and attachment & 37 & $3.5 \%$ \\
\hline
\end{tabular}

\section{Discussion}

4.1 Scientific Importance of this research

Breastfeeding save lives in all contexts - even more so in humanitarian and fragile contexts. The protection, promotion and support of breastfeeding are among the most effective interventions to prevent child morbidity and mortality in humanitarian contexts. (10). Exclusive breastfeeding for the first six months and continued breastfeeding, thereafter, may prevent $13 \%$ of under-five deaths, primarily from infections resulting in diarrhoea, pneumonia, and neonatal sepsis. (10) Non breastfed infants have a 14 times higher risk of dying from any cause compared to exclusively breastfed infants, a 15 times higher risk pneumonia and a 10 times higher risk of diarrhoea. (11) Furthermore, the global impact of breastfeeding on maternal health is significant, with a reduction in the risk of breast cancer and potential to prevent 20,000 deaths (12). In environments where access to safe water is limited and food insecurity is a significant issue for families, exclusive breastfeeding for new-borns and infants is a lifesaving public health intervention. It is important to ensure that additional studies are conducted in similar contexts, to ensure that findings are reflected in practical measures to protect the lives of the youngest and most vulnerable in humanitarian settings, whilst providing the needed support for pregnant women and mothers.

This assessment provided new, updated information on the breastfeeding practices of the most vulnerable communities in the Gaza strip. Prior to this study the most recent data was the Multiple Indicator Cluster Survey of 2014 (9).

This study's findings highlight the potential role that midwives in humanitarian contexts, who have an integral role in the initiation, protection promotion and support of breastfeeding practices. This is particularly relevant in conflict settings, where the need for psychosocial and mental support for pregnant and postnatal women with babies is significant, given the continuous challenge of giving birth and raising children in such a difficult and unsafe environment.

The findings confirm the need to: a) ensure a concerted effort to fully adapt and implement the recommendations of the IYCF-E operational guidance (24); b) review and possibly improve the pre-service training for midwives and other health professionals who provide antenatal, intrapartum and post-natal care in relation to the protection, promotion and support of breastfeeding using recommended WHO modules (25); and scale up and exert efforts to ensure hospital and health facilities providing maternity services fully comply with the WHO/UNICEF Baby Friendly hospital (26).

For the vulnerable population of the Gaza strip, it is imperative to support midwives to provide skilled support at birth (both vaginal and caesarean) thus ensuring breastfeeding initiation. If breastfeeding is practiced in the Gaza strip as per recommendations, there will be increased protection for common childhood diseases such as diarrhoea and ARI, whilst ensuring that the available resources are used by the household to increase access to a diverse and appropriate diet for the mother and the other members of the family. Having skilled midwives providing lactation support and counselling is certainly recommended in all contexts, particularly humanitarian settings and fragile environments like the Gaza strip.

The findings of the study were disseminated and validated by the members of the Nutrition Working Group and were presented to national and international organizations working in the Gaza strip during a workshop in May 2019. Additional ad hoc presentations were provided to different sectors including Health, WASH, Food Security, Livelihoods and Protection from June to August 2019.

Finally, study findings illustrating the immediate need to focus on practical, focussed interventions aiming at the most vulnerable were shared and presented with the Humanitarian Country Team (lead by UN OCHA), UN agencies and the organizations and donors contributing and supporting the humanitarian response. This guided strategic discussion and initial direction with UN OCHA and relevant UN partners. The study findings ensure that the protection, promotion and support of breastfeeding focusing on the most vulnerable populations in the Gaza strip, will receive more attention, including financial resources. At the same time the findings of the study were used to inform the revision and update of the Humanitarian Needs Overview for 2020 and the Humanitarian Response Plan 2019-2020 providing additional windows of opportunity for resources for an integrated solution to the issues presented.

\subsection{Challenges to Research}

Security

The security situation in the Gaza strip is unpredictable and escalation of conflicts is always a possibility. This was a major consideration during the design and planning phase of the study, and plans were made to ensure successful completion, even in the event of potential security situations. Contingency plans included the identification of back up areas to be covered and/or the reduction of the sample size requirements were also included.

Political Situation 
The current political situation in the Gaza strip, exemplified by a strict set of requirements limiting study coverage and information to be collected by the Ministry of Interior in Gaza, was a critical challenge faced during the study. The Ministry of Interior reviewed the proposed questionnaire, as per local policy and requested that questions on socio-economic status and mental health be removed. This did not allow for an analysis of the results linking infant feeding practices and psychological condition of the caregiver, which, in a context like the Gaza strip, could be remarkable.

Capacity

Due to the conflict and travel restrictions within the Gaza strip, many professionals and health workers (including midwives) are not able to access regular trainings and capacity building opportunities. This is clearly identified as a major gap and highlighted the need to strengthen local capacity in relation to nutrition assessments and implementation using local capacities and resources. This is relevant to this study's findings, with associated challenges when finalising study design and methodology, data collection and the quality of some aspects of the study.

\subsection{Research Strategies}

The following strategies and actions were deployed to address most of the challenges encountered:

- Integration and linkage with security officers of leading organizations was essential, during the design, planning and execution of all the activities. During the data collection phase, it was continuous and provided valuable information that lead to the temporary interruption of the data collection due to a rapid escalation of the conflict affecting the selected areas. In a volatile and unpredictable context like the Gaza strip, daily movements of teams and large groups must be cleared by relevant officers, mostly considering the locations of destination.

- Coordination with authorities and communities, a requirement for the issuance of the needed formal endorsements and clearances by the governing authorities as well ensuring full engagement and support from the participating communities. The Ministry of Interior in Gaza (under the Hamas leadership) required the review and clearance of the design and questionnaires. Several questions were removed as per their recommendations, that called for a revision of the questionnaire and of the objectives of the study.

- Supportive supervision was provided during the whole data collection with daily debriefing with teams, with the support of the leading organizations and to the extent possible international consultant. However, considering the escalation of the hostility during this period, this was reduced drastically and limited to Phone SMS based follow ups and updates. Teams were asked to communicate locations, challenges and progress, regularly during the day, to ensure their safe and smooth progress in the data collection.

- Allocation of buffer days and geographical areas in lieu of days lost. Having a contingency plan for similar exercises in the context of the Gaza strip is crucial. Due to the potential for escalation of conflict in selected areas, this extra allocation in relation to logistical support and timeframe mitigated any major impact on the actual timely conclusion of the assessment. Data collection was halted for 2 days due to the escalation of the conflict that affected the selected areas, and the buffer days were used to complete the data collection. Allocation of extra days meant ensuring that budget, cars, and other logistical support was available in a timely manner and did not require extra approvals and procedures.

- In country and remote assistance to the data collection teams were provided by all the agencies involved during the data collection but mostly during data processing and analysis. The lead researcher could not visit all the areas and during the potential escalation of the conflict could not visit the field and support the data collection teams

\section{Conclusions}

The study in Gaza highlights the importance of supporting research in protracted crisis settings. It proves that it is possible to conduct research in complex humanitarian environments. The value of this study is linked to its context and the living conditions of the most vulnerable communities in the Gaza strip. Lessons learned in this research include the importance of a) allocating additional research time, to account for potential interruption, due to the volatile situation b) daily coordination with security officers to ensure safe access to target localities c) identification of extra sites, in case escalation of conflicts do not allow for access to pre-selected localities and d) training of additional enumerators to fill up for potential gaps due to the conflict escalation and e) negotiation with the authorities to ensure compliance with the requirements. Finally, it was observed that there is a general need for health and nutrition workers to increase their knowledge and skills on nutrition assessments.

Despite the documented challenges, this research has highlighted the urgent need to review midwives' current skills and availability to provide breastfeeding support, including the timely initiation of breastfeeding and lactation support. Indeed, the protracted emergency settings may have limited midwives' ability to access professional updates and on the job capacity building opportunities. Implementing partners must address this gap in knowledge and skill as a matter of urgency.

The role of midwives in supporting women to improve their breastfeeding practices has several important effects on maternal health and wellbeing and proven positive effects for children. In humanitarian contexts such as the Gaza strip, the initiation and continuation of effective breastfeeding has lifesaving impacts. This study raises alerts humanitarian agencies on the importance of the need to invest more in evidence-based interventions and the capacity of midwives in humanitarian contexts. If this need is met, midwives' effective support of improved breastfeeding practices will contribute to reducing pressure on Gaza's public health system, currently stretched in a fragile and constant crisis setting.

\section{Abbreviations}

1. ARI: Acute Respiratory Infection

2. CS: Caesarean 
3. ENA: Emergency Nutrition Assessment

4. IYCF: Infant and Young Child Feeding

5. IYCF-E: Infant and Young Child Feeding in emergencies

6. KAP: Knowledge, Attitudes and Practices MEAL: Monitoring, Evaluation, Accountability and Learning

7. MICS: Multi Indicator Cluster Survey

8. OCHA: United Nations Office for the Coordination of Humanitarian Affairs

9. oPt: Occupied Palestinian Territory

10. SCl: Save the Children International Occupied Palestinian Territory

11. SMART: Standardized Monitoring and Assessment of Relief and Transitions

12. UNICEF: United Nations Children's Funds

13. UN: United Nations

14. UK: United Kingdom

15. WASH: Water, Sanitation and Hygiene

16. WFP: World Food Programme

\section{Declarations}

\section{Ethics approval and consent to participate}

The study was part of a larger assessment and was agreed by the Nutrition Working Group, a sub working group of the Health Cluster in the Gaza strip. All assessments and data collection exercises require the approval of the Ministry of Interior (MOI) in the Gaza strip. The MOI is the clearing house and approving agencies for all data collection and assessments in the Gaza strip. The MOI reviewed, commented and approved the conduct of the study, with some modifications in the questionnaire and in the design. No other ethical reviews were sought by the Nutrition Working Group and its members. Additional ethical reviews were waived because the $\mathrm{MOI}$ approved the conduct of the assessment, and the assessment was endorsed by the members of the Health Cluster, like the $\mathrm{MOH}, \mathrm{UN}$ agencies and national and international partners. The assessment was implemented also to inform the development of the the Humanitarian Needs Overview and the Humanitarian Response Plan for the Gaza strip.

The final approval was given verbally after the changes were done accordingly. The Ministry of Interior reviewed the questionnaire and the methodology including the verbal approval by the respondents to participate in the study.

All the respondents, after explaining the purpose of the study were asked if they agreed to participate in the survey. Their verbal consent was recorded in the Kobo survey tool.

\section{Consent for publication}

Not applicable

\section{Availability of data and materials}

The datasets generated and/or analysed during the current study are not publicly available due to the specific operational context but are available from the corresponding author on reasonable request and upon approval from the leading agencies.

\section{Competing interests}

The authors declare that they have no competing interests

\section{Funding}

SCl oPt, WFP and UNICEF contributed to the funding of the study. All the three agencies participated in the design, data collection, analysis and interpretation of the study.

\section{Authors' contributions}

1. Al contributed in the design, lead the analysis, interpretation and writing of the article

2. EM contributed in the interpretation and had a major role in the writing of the article

3. SM had a major role in the design, data collection and analysis of the data, participated in the finalization of the article

4. JL lead the design of the study and the data collection, contributed to the analysis of the study

5. NN had a major role in the design, data collection and analysis of the data, participated in the drafting and finalization of the article

All authors read and approved the final manuscript

Acknowledgements 
The assessment is the result of efforts and investments of different partners at every level, country, regional and global. We would like to recognise the important and strategic support from all the members of the Nutrition Working Group the Health Cluster lead and in particular the head of agencies Jennifer Moorehead, Country Director SCI oPt, Genevieve Boutin, UNICEF Special Representative and Stephen Kearney, WFP Representative and Country Director.

A major thanks also for their expertise and contribution to the following Selena Bajraktarevic, UNICEF Chief of Health and Nutrition, Dr Younis Awadallah, UNICEF Health Specialist and Amani Jouda, UNICEF Early Childhood Development Officer. Nitesh Patel, WFP Regional Nutrition Advisor, Samah Helou, WFP Programme Policy Officer, Salah Lahham, WFP Vulnerability Analysis and Mapping Officer and Ruba Shanti, WFP Vulnerability Analysis and Mapping Associate

\section{References}

1. UN OCHA, Palestine, Humanitarian Needs Overview, 2019. https://www.ochaopt.org/content/humanitarian-needs-overview-2019 accessed May 2019

2. Food Security Cluster Palestine. Socio Economic Food Security Survey 2018. Preliminary Results. Jerusalem,2018. https://fscluster.org/state-ofpalestine/document/sefsec-2018-food-security-analysis. Accessed July 2019

3. Web Report. https://www.unrwa.org/activity/health-gaza-strip accessed June 2019

4. Health conditions in the occupied Palestinian territory, including east Jerusalem, and in the occupied Syrian Golan. Geneva 2019. https://apps.who.int/gb/ebwha/pdf_files/WHA72/A72_33-en.pdf accessed July 2019

5. Health and Nutrition Profile, Palestine. https://www.unicef.org/sop/what-we-do/health-and-nutrition accessed June 2019

6. Ministry of Health Palestine. Nutrition Surveillance Report, 2016. Ramallah, West Bank https://site.moh.ps/Content/Books/ZxRcynmiUofNqt66u4CrHRgmJR6Uv7z77srjjIEAho6xnz5V3rgLTu_RhO7xf2j2VusNilvWkjwp84yXHLdGleB97gKrHHI5iž accessed July 2019

7. Palestinian Central Bureau of Statistics. Census 2017. http://www.pcbs.gov.ps/Downloads/book2362.pdf access July 2019

8. ENA software https://smartmethodology.org/survey-planning-tools/smart-emergency-nutrition-assessment/ accessed July 2019

9. UNICEF and PCBS. Multiple Indicator Cluster Survey State of Palestine, 2014. http://mics.unicef.org/news_entries/32 accessed June 2019

10. Jones et al. How many child deaths can we prevent this year? THE LANCET • Vol $362 \cdot$ July 5,2003

11. Black RE, Allen LH, Bhutta ZA, Caulfield LE, de Onis M, Ezzati M, \& al, for the Maternal and Child Undernutrition Study Group* Maternal and child undernutrition: global and regional exposures and health consequences. Lancet 2008; 371: 243-60

12. Victora $\mathrm{G}$ et al. Breastfeeding in the 21 st century: epidemiology, mechanisms, and lifelong effect. The Lancet, Vol 387 January 30,2016

13. Jacobsen et al. Breastfeeding status as a predictor of mortality among refugee children in an emergency situation in Guinea-Bissau, Tropical Medicine and International Health 2003. Volume 8 no 11 pp 992-996 November 2003

14. Anderson $\mathrm{N}$ et al. Breast-feeding in a complex emergency: four linked cross-sectional studies during the Bosnian conflict. Volume 13 , Issue 12 December 2010, pp. 2097-2104. Published online by Cambridge University Press: 25 June 2010

15. Creek T, Kim A, Lu L, . Role of infant feeding and HIV in a severe outbreak of diarrhoea and malnutrition among young children, Botswana, 2006 Paper presented at: 14th Conference on Retroviruses and Opportunistic Infections; February 3-7, 2007; Los Angeles, CA

16. B. Hipgrave, F. Assefa, A. Winoto, and S. Sukotjo, "Donated breast milk substitutes and incidence of diarrhoea among infants and young children after the May 2006 earthquake in Yogyakarta and Central Java.," Public Health Nutr., vol. 15, no. 2, pp. 307-15, Feb. 2012.

17. Ishii et al. Factors Associated with Infant Feeding Methods after the Nuclear Power Plant Accident in Fukushima: Data from the Pregnancy and Birth Survey for the Fiscal Year 2011 Fukushima Health Management Survey. Matern Child Health J. 2016; 20: 1704-1712.

18. Slade AC et al. Shaken but not broken: Supporting breastfeeding women after the 2011 Christchurch New Zealand earthquake. Breastfeed Rev. 2015 Nov;23(3):7-13.

19. DeYoung SE et al. The Effect of Mass Evacuation on Infant Feeding: The Case of the 2016 Fort McMurray Wildfire. Matern Child Health. 2018 Jul 27. doi: 10.1007/s10995-018-2585-z. [Epub ahead of print]

20. Ip et al. A summary of the Agency for Healthcare Research and Quality's evidence report on breastfeeding in developed countries. Breastfeed Med.2009 Oct;4 Suppl 1:S17-30. doi: 10.1089/bfm.2009.0050.

21. Kathryn G. Dewey and Sandra L. Huffman . Maternal, infant, and young child nutrition: Combining efforts to maximise impacts on child growth and micronutrient status. Food and Nutrition Bulletin, vol. 30, no. 2 @ 2009 (supplement), The United Nations University

22. Horta BL, Bahl R, Martinés JC, Victora CG. Evidence on the long-term effects of breastfeeding: Systematic reviews and meta-analyses. Geneva: World Health Organization, 2007.

23. Alison Mc Fadden et al, Support for healthy breastfeeding mothers with healthy term babies. Cochrane Systematic Review - Intervention Version published: 28 February 2017

24. ENN, IFE Core Group. Infant and Young Child Feeding in Emergencies. Operational Guidance for Emergency Relief Staff and Programme Managers. Version 3.0 October 2017. https://www.ennonline.net/attachments/3127/Ops-G_English_04Mar2019_WEB.pdf accessed June 2019

25. Infant and Young Child Feeding. Model Chapter for Textbooks for medical students and allied professions. Geneva 2019. https://www.who.int/maternal_child_adolescent/documents/9789241597494/en/ accessed June 2019

26. WHO UNICEF Baby Friendly Hospital Initiative recommendations. Geneva 2018. https://www.who.int/nutrition/bfhi/ten-steps/en/ accessed May 2019. 